OPEN ACCESS

Edited by:

Robin Lemmens,

University Hospitals Leuven, Belgium

Reviewed by:

Else Charlotte Sandset,

Oslo University Hospital, Norway

Jukka Putaala

Helsinki University Central

Hospital, Finland

*Correspondence:

Angelos Sharobeam

angelos.sharobeam@mh.org.au

Specialty section:

This article was submitted to

Stroke,

a section of the journal

Frontiers in Neurology

Received: 15 September 2020 Accepted: 23 November 2020 Published: 08 December 2020

Citation:

Sharobeam A, Churilov L, Parsons M,

Donnan GA, Davis SM and Yan B (2020) Patterns of Infarction on MRI in Patients With Acute Ischemic Stroke and Cardio-Embolism: A Systematic Review and Meta-Analysis.

Front. Neurol. 11:606521

doi: 10.3389/fneur.2020.606521

\section{Patterns of Infarction on MRI in Patients With Acute Ischemic Stroke and Cardio-Embolism: A Systematic Review and Meta-Analysis}

\author{
Angelos Sharobeam ${ }^{1 *}$, Leonid Churilov ${ }^{1,2,3}$, Mark Parsons ${ }^{1,4,5,6}$, Geoffrey A. Donnan ${ }^{1,2}$, \\ Stephen M. Davis ${ }^{1,2}$ and Bernard Yan ${ }^{1,2}$ \\ ${ }^{1}$ Melbourne Brain Centre at the Royal Melbourne Hospital, Parkville, VIC, Australia, ${ }^{2}$ Faculty of Medicine, Dentistry and \\ Health Sciences, The University of Melbourne, Parkville, VIC, Australia, ${ }^{3}$ Florey Institute of Neuroscience and Mental Health, \\ The University of Melbourne, Parkville, VIC, Australia, ${ }^{4}$ South Western Sydney Clinical School, The University of New South \\ Wales, Liverpool, NSW, Australia, ${ }^{5}$ Department of Neurology, Liverpool Hospital, Liverpool, NSW, Australia, ${ }^{6}$ The Ingham \\ Institute, Liverpool, NSW, Australia
}

Background: Cardioembolic strokes are common however atrial fibrillation, the most common cause, is often asymptomatic and difficult to detect. There is evidence that infarct topography and volume on magnetic resonance imaging may be associated with specific stroke etiologies.

Aim: A systematic review and meta-analysis were undertaken to summarize the available evidence on the association between stroke etiology, infarct topography, and volume.

Methods: A systematic review was conducted using Medline (OVID), Embase (OVID), and PubMed databases. Hand searches of the gray literature and of reference lists in relevant articles were also performed. A quality assessment was undertaken, based on the STROBE checklist. For each study, the number of patients with and without a CE source of stroke and infarct topography was collected and outcomes presented as odds ratios (OR) with $95 \% \mathrm{Cl}$ and $p$-values.

Results: Four thousand eight hundred and seventy-three patients with ischemic stroke were included, of whom 1,559 were determined to have a CE source. Bilateral infarcts (OR 3.41; 95\% Cl 2.20-5.29; $p<0.0001$ ) and multiple territory infarcts (OR 1.57; 95\% $\mathrm{Cl} 1.12-2.21 ; p=0.009$ ) were more common in patients with a CE source of stroke, than patients without a CE source. Lacunar infarcts (OR 0.49; 95\% Cl 0.31-0.80; $p=$ 0.004 ) were more likely to occur in patients without a CE source. No significant difference between the frequency of multiple infarcts (OR 0.96; 95\% $\mathrm{Cl} 0.57-1.61 ; p=0.87$ ) anterior circulation (OR 1.45; 95\% Cl 0.83-2.53; $p=0.19$ ) or posterior circulation infarcts (OR $1.06 ; 95 \% \mathrm{Cl} 0.72-1.57 ; p=0.75)$, between the two groups were identified. Three out of four studies examining volume, found a significant association between increased infarct volume and CE source of stroke. A sensitivity analysis with cryptogenic and undetermined stroke sources assumed to be cardioembolic, did not alter the associations observed. 
Conclusion: The findings of this systematic review and meta-analysis are broadly consistent with previous literature and provide more robust evidence on the association between infarct topography, volume and stroke etiology. Our findings may assist with refining cardiac investigations for patients with cryptogenic stroke, based on infarct topography.

Keywords: stroke, cardio-embolism, magnetic resonance imaging, topography, volume

\section{INTRODUCTION}

Ischemic stroke is a common cause of morbidity globally, with over 116 million years of healthy life lost each year due to stroke related death and disability (1). Cardioembolic (CE) sources form the underlying etiology in $20-25 \%$ of cases (2). Atrial fibrillation $(\mathrm{AF})$, a common cause of CE stroke, may be asymptomatic in one third of patients and hence may go undetected (3). This has major implications for management, given the proven benefit of anticoagulation $(4,5)$. Current diagnostic techniques for $\mathrm{AF}$ detection are limited by low detection rate, particularly with non-invasive techniques such as Holter monitoring and wearable devices (6). Cost effectiveness if also a factor. The test with the highest detection rate, an implantable cardiac monitor (ICM), is not cost-effective with the incremental cost-effectiveness ratio (ICER) at $£ 17,175$ per quality of life year (QALY) gained, compared to 24 -h cardiac telemetry (7).

Previous studies have examined factors, which predict the presence of $\mathrm{AF}$ in patients with ischemic stroke. Older age, atrial cardiopathy (8), and a prolonged PR interval on ECG, are independently associated with increased incidence of AF (911). Serum biomarkers, such as brain natriuretic peptide, have been associated with future development of paroxysmal $\mathrm{AF}$ in patients with ischemic stroke $(12,13)$. Thrombus histopathology has also been associated with stroke etiology, with CE source strokes more likely to be fibrin rich and erythrocyte poor (14, 15). In addition to findings from cardiac and pathology testing, there is evidence to suggest that a CE stroke etiology may be associated with topographical infarct patterns on magnetic resonance imaging (MRI).

Multi-territory MRI-DWI lesion topography is a common finding in CE strokes (16-18). In a large study by Chung et al. (17), multi-territory infarction was more prevalent amongst those with a CE etiology (representing $44.2 \%$ of all multi-territory infarctions). A number of other studies have demonstrated that a large proportion of patients with multi-territory infarction will have a CE source $(19,20)$. Akhtar et al. (19), in a systematic review of simultaneous infarcts in multiple territories, revealed that simultaneous infarcts in the anterior and posterior circulation, are more likely to be associated with AF than other etiologies such as large artery atherosclerosis (LAA). Such infarcts had an underlying CE source in two-thirds of cases.

A study by Yushan et al. (16) using MRI-DWI imaging demonstrated that $30 \%$ of patients with detected AF had bilateral infarcts at presentation, significantly higher than the $5.5 \%$ of the control cohort. Studies looking at anterior circulation infarcts did not show a clear predilection for $\mathrm{CE}$ stroke as opposed to other stroke etiologies (21-23). A study by Rizos et al., utilizing lesion mapping techniques, showed a higher probability of CE infarcts in the right insula compared to other regions (24).

A number of studies (25-27) examining infarct patterns in the posterior circulation revealed LAA was the most likely etiology, however CE etiologies were implicated in $20-30 \%$ of cases. In contrast, Chung et al. (17) showed that $60 \%$ of superior cerebellar artery infarcts have an underlying CE source. Schiphorst et al. (28), however, showed that small obliquely oriented cortical cerebellar infarcts have a higher risk of future diagnosis of $\mathrm{AF}$, compared to other cerebellar infarct subsets.

Larger infarct volumes have been associated with CE source of stroke, in multiple studies (24, 29-31). In addition to infarct size, subsequent infarct growth, and hemorrhagic transformation are also strongly predictive of underlying $\mathrm{CE}$ source. In a secondary analysis of the EPITHET trial by $\mathrm{Tu}$ et al. (29), patients with definite AF had significantly greater infarct growth, larger infarcts, more frequent parenchymal hematoma grade hemorrhagic transformation, worse functional outcomes and higher mortality compared to patients with no AF. An analysis of infarct volume by Rizos et al. (24) likewise showed larger volumes in patients with both new and established AF.

\section{Hypothesis/Study Question}

In patients with acute ischemic stroke, infarct pattern and volume on MRI will differ between patients with and without a CE source.

\section{METHODS}

\section{Literature Search}

A systematic review was performed according to PRISMA guidelines (32) and registered with the PROSPERO register of systematic reviews (Registration ID CRD42020169479). The search strategy (Supplementary Material 1) included a combination of controlled synonyms and vocabularies from Medical Subject Headings (MeSH) and EmTree covering stroke, infarction, pattern, topography, distribution, atrial fibrillation, cardioembolism, magnetic resonance imaging, and diffusion weighted imaging. The search was conducted using Medline (OVID), Embase (OVID), and PubMed databases using a combination of truncations and Boolean operators. Hand searches of the gray literature and of reference lists in relevant articles were also performed. Two co-authors reviewed the abstracts and selected the included studies (AS, BY). The literature search excluded reviews, editorials, and conference abstracts. The search strategy is summarized in Figure $\mathbf{1}$. 


\section{Records identified through database searching $(\mathrm{n}=623)$}

\section{Additional records identified} through hand searching $(\mathrm{n}=22)$

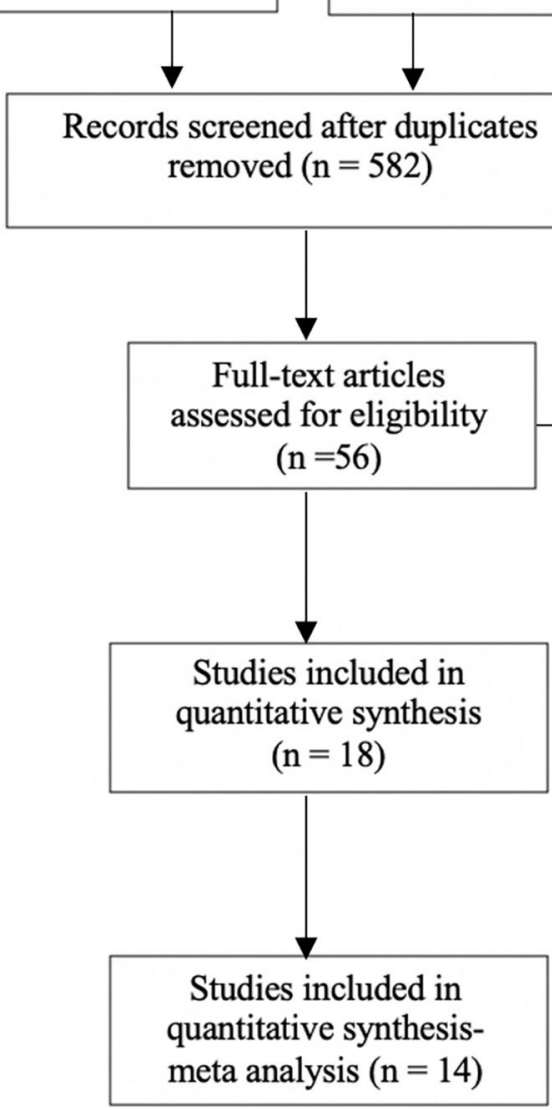

FIGURE 1 | Search strategy for meta-analysis. Full text articles analyzed, may have more than one reason for exclusion. Eighteen articles were included, with four excluded from the meta-analysis due to analysis of volume only.

\section{Inclusion Criteria}

A quality assessment of all full text articles selected was undertaken (Supplementary Table 1 in Supplementary Material 2) based on The Strengthening the Reporting of Observational Studies in Epidemiology (STROBE) checklist and Joanna Briggs Institute checklists for crosssectional studies $(33,34)$. To be included in the meta-analysis, all studies were required to meet the following minimum criteria:

1. All patients had an MRI with diffusion weighted imaging (DWI) sequence performed.

2. Infarct topography is clearly defined.

3. The criteria for a stroke categorized as cardio-embolic is clearly defined. For example, using the TOAST criteria (35).

4. All included patients have been investigated for cardioembolic sources, at a minimum with baseline ECG and $24 \mathrm{~h}$ of cardiac telemetry plus echocardiography if no cause found.

5. A comparison group is available, i.e., cardioembolic vs. noncardioembolic strokes.
6. Background and objectives are clearly delineated.

7. Participant selection criteria are outlined.

8. Appropriate synthesis, outcome measures, methodology, and statistics.

9. Confounders and limitations are addressed.

\section{Data Extraction}

For each study, the total number of patients with CE stroke, the total number of stroke patients, the breakdown of stroke etiologies and topographical infarct patterns on MRI, were collected. Stroke etiology was dichotomized as being either CE or non-CE source. Where etiology was undetermined, the cause was assumed to be non-CE. A sensitivity analysis with the opposite assumption, with undetermined etiology assumed to be $\mathrm{CE}$, was therefore performed which revealed no difference in the results (Supplementary Material 3). Topographical patterns analyzed were bilateral infarcts, multiple infarcts, multiple vascular territory infarcts, lacunar, anterior circulation, posterior 
circulation, internal watershed, and external watershed infarcts. Papers mentioning infarct volume, were also analyzed.

\section{Statistical Analysis}

Review Manager (RevMan) version 5.3 (Copenhagen: The Nordic Cochrane Center, The Cochrane Collaboration, 2014) was used to perform a meta-analysis of observational studies fulfilling the above criteria. The event rate for individual topographical patterns and 95\% confidence intervals (CI) were determined from each study. Each topographical pattern was analyzed separately. Outcomes are presented as odds ratios (OR) with 95\% CI and p-values. We used the Mantel-Haenszel implementation of the DerSimonian and Laird random-effect method implemented in RevMan (36). This implementation estimates the amount of between-study variation by comparing each study's result with a Mantel-Haenszel fixed-effect metaanalysis result (36). Heterogeneity of treatment effect across studies was evaluated by using the $I^{2}$ statistic, in which $I^{2}>$ $50 \%$ suggests substantial heterogeneity, as per the guide for interpretation of thresholds for $I^{2}$ from the Cochrane handbook chapter 10 (36). A $p<0.05$ was treated as indicative of statistical significance. Funnel plot asymmetry and Egger's test was used to illustrate the extent of small study publication bias (Supplementary Material 4).

\section{RESULTS}

The literature search yielded 632 articles. Following review of titles, abstracts, exclusion of conference abstracts, and review articles, 45 full text papers were considered for inclusion. Fourteen papers exploring topography fulfilled all inclusion criteria $(16,18,26,37-47)$ and were included in a meta-analysis.

A total of 4,873 patients with ischemic stroke were included, of whom 1,559 were determined to have a CE source. Bilateral infarcts (OR 3.41; 95\% CI 2.20-5.29; $p<0.0001$ ) and multiple territory infarcts (OR 1.57; 95\% CI $1.12-2.21 ; p=0.009$ ) were more common in patients with a CE source of stroke, than patients without a CE source. Lacunar infarcts (OR 0.49; 95\% CI $0.31-0.80 ; p=0.004)$ were more likely to occur in patients without a CE source. No significant difference between the frequency of multiple infarcts (OR 0.96; 95\% CI 0.57-1.61; $p=$ 0.87 ) anterior circulation (OR $1.45 ; 95 \%$ CI $0.83-2.53 ; p=0.19$ ) or posterior circulation infarcts (OR 1.06; 95\% CI $0.72-1.57$; $p$ $=0.75$ ), between the two groups were identified (Figures 2A-F). No significant heterogeneity was detected in the bilateral and multiple territory subgroups; however, the other subgroups did display significant heterogeneity between the included studies. Egger's test did not reveal any evidence of publication bias. Only one paper (39) analyzed watershed infarcts. Of 43 patients with watershed infarcts, 27 were classified as external watershed and 16 as internal watershed. In both cases, a non-CE etiology was more common than a CE etiology. Volume was not included in the meta-analysis, due to the low number of studies included and non-uniform reporting methods.

A sub-analysis of multiple territory and bilateral infarcts was performed on papers which also reported chronic infarcts in these territories. Only one paper reported on the presence of concurrent chronic infarcts (44). There were numerically more chronic multi-territory infarcts seen in the non-AF population $(15 / 83,18 \%)$ than the AF population $(2 / 21,9.5 \%)$, although this did not reach statistical significance.

\section{Infarct Volume}

Four papers meeting inclusion criteria also analyzed infarct volume (2, 4, 29-31). The median baseline volume in CE ranged from $8.3 \mathrm{ml}$ in $\mathrm{Kim}$ et al. (30) to $22.5 \mathrm{ml}$ in $\mathrm{Tu}$ et al. (29) and the non-CE ranged from $3.0 \mathrm{ml}$ in $\mathrm{Kim}$ et al. (30) to $4.2 \mathrm{ml}$ in $\mathrm{Tu}$ et al. (29). In two out of three studies $(29,30)$, the baseline infarct volume with statistically significantly higher in CE compared to non-CE stroke. Rizos et al. (24) reported a mean baseline infarct volume of $41.2 \mathrm{ml}$, which was likewise significantly higher than patients with non-CE source of stroke $(15.1 \mathrm{ml})$.

\section{DISCUSSION}

We set out to determine differences between infarct pattern and volume. in patients with and without a CE source of stroke. The findings of our study indicate that larger infarct volumes and certain topographical patterns, including multiple territory and bilateral infarcts in any arterial territory, occur more commonly in patients with a CE source of stroke. Lacunar infarcts are more likely to be associated with a non-CE source. These findings support previous studies in which a correlation between a CE source and infarcts in multiple territories, including bilateral infarcts (16-20) was found. This association to CE stroke may be explained by atrial thrombus fragmentation, leading to infarcts in different arterial territories (46).

Although the causes of multi-territory infarcts do include non-CE etiologies (44), the association of non-CE etiologies with other patterns in our meta-analysis, particularly lacunar, would support the notion that non-CE source infarct topography is less likely to be multi-territory compared to CE source strokes.

The lack of association with either a CE or non-CE etiology in anterior circulation and posterior circulation strokes, may be due to the influence of multiple vascular risk factors, such as diabetes and ischemic heart disease influencing infarct location (48). One question that remains unanswered however is whether or not a CE source, such as AF, is more likely to determine topography, when two or more etiologies are considered equally likely. The results of our sensitivity analysis, which showed no difference in patterns, would suggest that intercurrent CE sources offer no additional weighting to the topography of the infarct.

Studies analyzing volume, all indicated that larger infarct volumes are more likely to be associated with a cardio-embolic source. This finding supports the findings of $\mathrm{Tu}$ (49), who postulated that larger infarct volumes were the result of poorer collateral circulation in patients with AF.

Strengths of our study include the definitions of topography and volume based on MRI alone, rather than CT, and the inclusion of a large number of patients $(4,873)$, which reduces the likelihood that topographical associations were due to chance alone. The comparison to other possible stroke sources and the criteria for minimum cardiac investigations, reduces the likelihood of a missed CE source. 

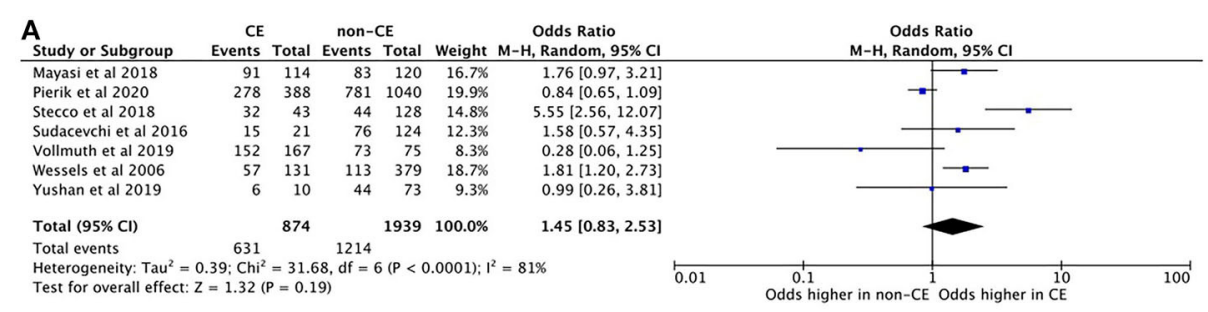

B

\begin{tabular}{l} 
B \\
Study or Subgroup \\
\hline Mayasi et al 2018 \\
Pierik et al 2020 \\
Stecco et al 2017 \\
Sudacevschi et al 2016 \\
Vollmuth et al 2019 \\
Wessels et al 2006 \\
Yushan et al 2019
\end{tabular}

CE non-CE Odds Ratio

Odds higher in non-CE Odds higher in CE

Total $(95 \% \mathrm{Cl})$

Total events 243

Heterogeneity: $\mathrm{Tau}^{2}=0.15 ; \mathrm{Chi}^{2}=16.03, \mathrm{df}=6(\mathrm{P}=0.01) ; \mathrm{I}^{2}=63 \%$

Test for overall effect: $Z=0.31(P=0.75)$

$\begin{array}{llllll}23 & 114 & 36 & 120 & 16.0 \% & 0.59(0.32,1.08)\end{array}$

$\begin{array}{rrrrrr}23 & 114 & 36 & 120 & 16.0 \% & 0.59[0.32,1.08] \\ 110 & 388 & 259 & 1040 & 23.4 \% & 1.19[0.92,1.55]\end{array}$

$\begin{array}{rrrrrr}10 & 388 & 259 & 1040 & 23.4 \% & 1.19[0.92,1.55]\end{array}$

$\begin{array}{rrrrrr}7 & 43 & 42 & 128 & 11.0 \% & 0.40[0.16,0.97] \\ 6 & 21 & 40 & 124 & 9.3 \% & 0.84[0.30,2.33]\end{array}$

$\begin{array}{rrrrrr}6 & 21 & 40 & 124 & 9.3 \% & 0.84[0.30,2.33]\end{array}$

$\begin{array}{llllll}29 & 131 & 48 & 379 & 17.9 \% & 1.52[0.84,2.75]\end{array}$

$1.96[1.18,3.27]$

$1.06[0.72,1.57]$

\begin{abstract}
$874 \quad 1939 \quad 100.0 \%$
\end{abstract}

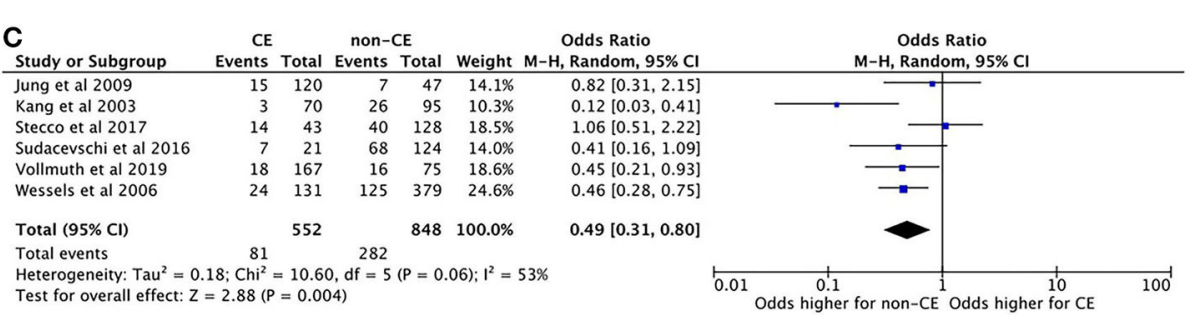

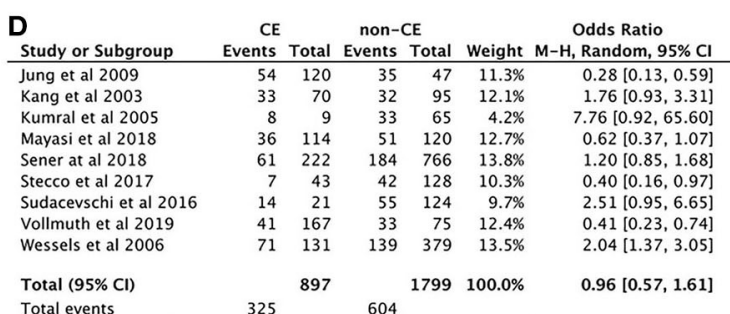

$\begin{array}{lcc}\text { Total events } & 325 & 604 \\ \text { Heterogeneity: } \mathrm{Tau}^{2}=0.48 ; \mathrm{Chi}^{2}=50.24, \mathrm{df}=8(\mathrm{P}<0.00001) & \left.\right|^{2}=84 \%\end{array}$

Test for overall effect: $Z=0.16(P=0.87)$

Odds Ratio

M-H, Random, $95 \% \mathrm{CI}$

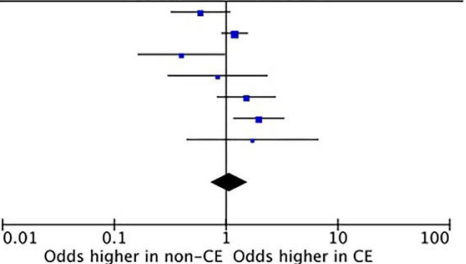

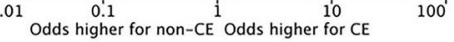

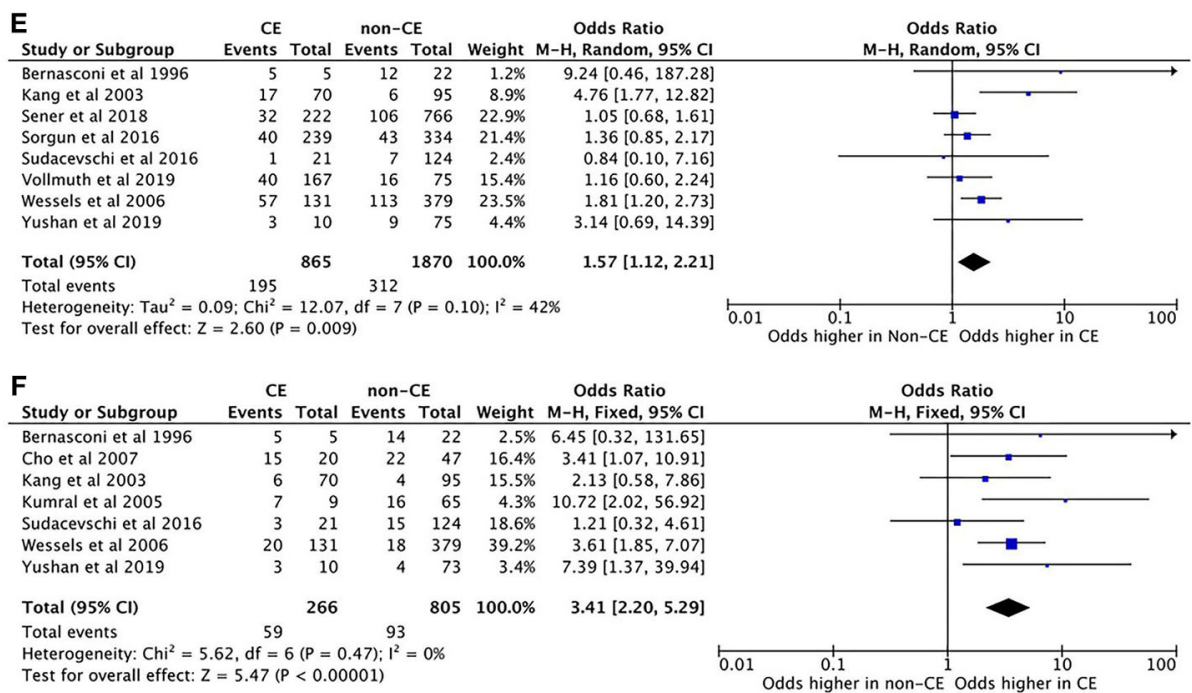

FIGURE 2 | (A) Forest plot showing the odds ratio of anterior circulation infarct topography in CE stroke, compared to non-CE stroke. (B) Forest plot showing the odds ratio of posterior circulation infarct topography in CE stroke, compared to non-CE stroke. (C) Forest plot showing the odds ratio of lacunar infarct topography in CE stroke, compared to non-CE stroke. (D) Forest plot showing the odds ratio of multiple infarcts in one or more arterial territories in CE stroke, compared to non-CE stroke. (E) Forest plot showing the odds ratio of infarcts in multiple large artery territories in CE stroke, compared to non-CE stroke. (F) Forest plot showing the odds ratio of bilateral hemisphere infarcts in CE stroke, compared to non-CE stroke. 
An urgent need exists for rapid, non-invasive, accurate tests for AF detection. There is an unanswered question regarding anticoagulation for cryptogenic stroke, particularly those with embolic stroke of undetermined source (ESUS). The findings of our study are pertinent given negative studies for anticoagulation is ESUS so far $(50,51)$. The results of our analysis suggest the cryptogenic source strokes offer no additional weighting to infarct source based on MRI topography. In line with recent literature $(52,53)$, this may in turn indicate that a large portion of cryptogenic strokes may actually be non-cardioembolic and therefore will not benefit from anticoagulation.

\section{LIMITATIONS}

There were a number of limitations to this study. First and foremost, in all studies, the etiology of ischemic stroke could not be determined with absolute certainty, as this would necessitate screening for all potential stroke risk factors in all patients. Although PRISMA guidelines were adhered to, there is a risk of selection and reporting bias, as all studies are observational and the majority retrospective in nature. Not all included studies, reported on all topographical patterns, hence comparison was made on available data. Infarct patterns were often common to multiple groups; for example, patients with multi-territory infarcts also had anterior or posterior circulation infarcts recorded. It was often not possible to determine the proportion of isolated infarcts in different circulations, as this was often not explicitly stated (with the exception of lacunar infarcts). Other confounders, such as anticoagulant and statin use, could not be accounted for. There was significant heterogeneity in a number of subgroups, likely reflecting the inclusion of studies with low patient numbers.

\section{CONCLUSIONS AND FUTURE DIRECTIONS}

This study has provided further evidence for associations between infarct volume, topography and CE stroke. Further large, long term prospective studies, with low cardiovascular risk

\section{REFERENCES}

1. Johnson CO, Nguyen M, Roth GA, Nichols E, Alam T, Abate D, et al. Global, regional, and national burden of stroke, 1990-2016: a systematic analysis for the Global Burden of Disease Study 2016. Lancet Neurol. (2019) 18:439-58. doi: 10.1016/S1474-4422(19)30034-1

2. O’Donnell MJ, Chin SL, Rangarajan S, Xavier D, Liu L, Zhang H, et al. Risk factors for ischemic and intracerebral haemorrhagic stroke in 22 countries (the INTERSTROKE study): a case-control study. Lancet. (2010) 376:11223. doi: 10.1016/S0140-6736(10)60834-3

3. Dilaveris PE, Kennedy HL. Silent atrial fibrillation: epidemiology, diagnosis and clinical impact. Clin Cardiol. (2017) 40:413-8. doi: 10.1002/clc.22667

4. Hart RG, Pearce LA, Aguilar MI. Meta-analysis: antithrombotic therapy to prevent stroke in patients who have nonvalvular atrial fibrillation. Ann Intern Med. (2007) 146:857-67. doi: 10.7326/0003-4819-146-12-200706190-00007

5. van Walraven C, Hart RG, Singer DE, Laupacis A, Connolly S, Petersen $\mathrm{P}$, et al. Oral anticoagulants vs aspirin in nonvalvular populations, are needed to demonstrate this link. The use of more sensitive methods for determining infarct topography and volume than human visual analysis, such as machine learning, may also improve our understanding of infarct etiology in future. The use of machine learning in stroke neuroimaging is already well-established, being utilized for determination of ischemic penumbra and large vessel occlusions (54). Emerging applications include the prediction of stroke onset time, functional outcomes following stroke, neurological deterioration, and hemorrhagic transformation (55). Future studies utilizing machine learning in infarct topography may offer additional, more accurate diagnostic tools for determination of stroke etiology. This may in turn lead to future anticoagulation trials in patients with high risk cardioembolic patterns, looking for stroke recurrence.

In the interim, this study may assist with refining cardiac investigations for patients with cryptogenic stroke, based on infarct topography.

\section{DATA AVAILABILITY STATEMENT}

The original contributions presented in the study are included in the article/Supplementary Materials, further inquiries can be directed to the corresponding author.

\section{AUTHOR CONTRIBUTIONS}

AS conceptualized the project and was responsible for primary writing of the text. LC and AS performed the statistical analysis and co-wrote the article methods. AS and BY selected and critically appraised included articles. SD, GD, LC, MP, and BY provided critical appraisal and editing of the manuscript. All authors approved the final version of the manuscript.

\section{SUPPLEMENTARY MATERIAL}

The Supplementary Material for this article can be found online at: https://www.frontiersin.org/articles/10.3389/fneur. 2020.606521/full\#supplementary-material

atrial fibrillation: an individual patient meta-analysis. JAMA. (2002) 288:2441-8. doi: 10.1001/jama.288.19.2441

6. Schnabel RB, Haeusler KG, Healey JS, Freedman B, Boriani G, Brachmann J, et al. Searching for atrial fibrillation poststroke: a white paper of the AF-SCREEN International Collaboration. Circulation. (2019) 140:183450. doi: 10.1161/CIRCULATIONAHA.119.040267

7. Diamantopoulos A, Sawyer LM, Lip GY, Witte KK, Reynolds MR, Fauchier $\mathrm{L}$, et al. Cost-effectiveness of an insertable cardiac monitor to detect atrial fibrillation in patients with cryptogenic stroke. Int J Stroke. (2016) 11:30212. doi: $10.1177 / 1747493015620803$

8. Kamel H, Bartz TM, Elkind MS, Okin PM, Thacker EL, Patton KK, et al. Atrial cardiopathy and the risk of ischemic stroke in the CHS (Cardiovascular Health Study). Stroke. (2018) 49:980-6. doi: 10.1161/STROKEAHA.117.020059

9. Thijs VN, Brachmann J, Morillo CA, Passman RS, Sanna T, Bernstein RA, et al. Predictors for atrial fibrillation detection after cryptogenic stroke: results from crystal AF. Neurology. (2016) 86:261-9. doi: 10.1212/WNL.0000000000002282 
10. Poli S, Diedler J, Hartig F, Gotz N, Bauer A, Sachse T, et al. Insertable cardiac monitors after cryptogenic stroke-a risk factor based approach to enhance the detection rate for paroxysmal atrial fibrillation. Eur J Neurol. (2016) 23:375-81. doi: 10.1111/ene.12843

11. Haeusler KG, Groschel K, Kohrmann M, Anker SD, Brachmann J, Bohm M, et al. Expert opinion paper on atrial fibrillation detection after ischemic stroke. Clin Res Cardiol. (2018) 107:871-80. doi: 10.1007/s00392-018-1256-9

12. Scheitz JF, Erdur H, Haeusler KG, Audebert HJ, Roser M, Laufs U, et al. Insular cortex lesions, cardiac troponin, and detection of previously unknown atrial fibrillation in acute ischemic stroke: insights from the troponin elevation in acute ischemic stroke study. Stroke. (2015) 46:1196201. doi: 10.1161/STROKEAHA.115.008681

13. Rodríguez-Yáñez M, Arias-Rivas S, Santamaría-Cadavid M, Sobrino T, Castillo J, Blanco M. High pro-BNP levels predict the occurrence of atrial fibrillation after cryptogenic stroke Neurology. (2013) 81:4447. doi: 10.1212/WNL.0b013e31829d8773

14. Sporns PB, Hanning U, Schwindt W, Velasco A, Minnerup $\mathrm{J}$, Zoubi $\mathrm{T}$, et al. Ischemic stroke: what does the histological composition tell us about the origin of the thrombus? Stroke. (2017) 48:2206-10. doi: 10.1161/STROKEAHA.117.016590

15. Maekawa K, Shibata M, Nakajima H, Mizutani A, Kitano Y, Seguchi M, et al. Erythrocyte-rich thrombus is associated with reduced number of maneuvers and procedure time in patients with acute ischemic stroke undergoing mechanical thrombectomy. Cerebrovasc Dis Extra. (2018) 8:3949. doi: 10.1159/000486042

16. Yushan B, Tan BYQ, Ngiam NJ, Chan BPL, Luen TH, Sharma VK, et al. Association between bilateral infarcts pattern and detection of occult atrial fibrillation in Embolic Stroke of Undetermined Source (ESUS) in patients with Insertable Cardiac Monitor (ICM). J Stroke Cerebrovasc Dis. (2019) 28:2448-52. doi: 10.1016/j.jstrokecerebrovasdis.2019.06.025

17. Chung JW, Hyun S, Kim N, Kim WJ, Park JH, Ko Y, et al. Trial of ORG 10172 in Acute Stroke Treatment (TOAST) classification and vascular territory of ischemic stroke lesions diagnosed by diffusion-weighted imaging. J Am Heart Assoc. (2014) 3:e001119. doi: 10.1161/JAHA.114.001119

18. Mayasi Y, Helenius J, McManus DD, Goddeau RP Jr, Jun-O'Connell $\mathrm{AH}$, Moonis $\mathrm{M}$, et al. Atrial fibrillation is associated with anterior predominant white matter lesions in patients presenting with embolic stroke. $J$ Neurol Neurosurg Psychiatry. (2018) 89:6-13. doi: 10.1136/jnnp-2016-315457

19. Akhtar T, Shahjouei S, Zand R. Etiologies of simultaneous cerebral infarcts in multiple arterial territories: a simple literature-based pooled analysis. Neurol India. (2019) 67:692-5. doi: 10.4103/0028-3886.263244

20. Depuydt S, Sarov M, Vandendries C, Guedj T, Cauquil C, Assayag P, et al. Significance of acute multiple infarcts in multiple cerebral circulations on initial diffusion weighted imaging in stroke patients. J Neurol Sci. (2014) 337:151-5. doi: 10.1016/j.jns.2013.11.039

21. Kang SY, Kim JS. Anterior cerebral artery infarction: stroke mechanism and clinical-imaging study in 100 patients. Neurology. (2008) 70:238693. doi: 10.1212/01.wnl.0000314686.94007.d0

22. Bogousslavsky J, Regli F. Anterior cerebral artery territory infarction in the lausanne stroke registry. clinical and etiologic patterns. Arch Neurol. (1990) 47:144-50. doi: 10.1001/archneur.1990.00530020040012

23. Sato S, Toyoda K, Matsuoka H, Okatsu H, Kasuya J, Takada T, et al. Isolated anterior cerebral artery territory infarction: dissection as an etiological mechanism. Cerebrovasc Dis. (2010) 29:170-7. doi: 10.1159/000262314

24. Rizos T, Bartsch AJ, Johnson TD, Dittgen F, Nichols TE, Malzahn U, et al. Voxelwise distribution of acute ischemic stroke lesions in patients with newly diagnosed atrial fibrillation: trigger of arrhythmia or only target of embolism? PLoS ONE. (2017) 12:e0177474. doi: 10.1371/journal.pone.0177474

25. Amarenco P, Levy C, Cohen A, Touboul PJ, Roullet E, Bousser MG. Causes and mechanisms of territorial and nonterritorial cerebellar infarcts in 115 consecutive patients. Stroke. (1994) 25:105-12. doi: 10.1161/01.STR.25.1.105

26. Kumral E, Kisabay A, Atac C, Calli C, Yunten N. Spectrum of the posterior inferior cerebellar artery territory infarcts. clinicaldiffusion-weighted imaging correlates. Cerebrovasc Dis. (2005) 20:370-80. doi: 10.1159/000088667

27. Kumral E, Kisabay A, Ataç C. Lesion patterns and etiology of ischemia in superior cerebellar artery territory infarcts. Cerebrovasc Dis. (2005) 19:283-90. doi: $10.1159 / 000084496$
28. Schiphorst AT, Tatu L, Thijs V, Demattei C, Thouvenot E, Renard D. Small obliquely oriented cortical cerebellar infarctions are associated with cardioembolic stroke. BMC Neurol. (2019) 19:100. doi: 10.1186/s12883-019-1328-0

29. Tu HT, Campbell BC, Christensen S, Desmond PM, De Silva DA, Parsons MW, et al. Worse stroke outcome in atrial fibrillation is explained by more severe hypoperfusion, infarct growth, and hemorrhagic transformation. Int $J$ Stroke. (2015) 10:534-40. doi: 10.1111/ijs.12007

30. Kim YD, Hong HJ, Cha MJ, Nam CM, Nam HS, Heo JH. Determinants of infarction patterns in cardioembolic stroke. Eur Neurol. (2011) 66:14550. doi: 10.1159/000330563

31. Jung JM, Kwon SU, Lee JH, Kang DW. Difference in infarct volume and patterns between cardioembolism and internal carotid artery disease: focus on the degree of cardioembolic risk and carotid stenosis. Cerebrovasc Dis. (2010) 29:490-6. doi: 10.1159/000297965

32. Moher D, Liberati A, Tetzlaff J, Altman DG, The PRISMA Group. Preferred Reporting Items for Systematic Reviews and Meta-Analyses: the PRISMA statement. PLoS Med. (2009) 6:e1000097. doi: 10.1371/journal.pmed.1000097

33. von Elm E, Altman DG, Egger M, Pocock SJ, Gotzsche PC, Vandenbroucke JP. The Strengthening the Reporting of Observational Studies in Epidemiology (STROBE) statement: guidelines for reporting observational studies. Ann Intern Med. (2007) 147:57377. doi: 10.7326/0003-4819-147-8-200710160-00010

34. Moola S, Munn Z, Tufanaru C, Aromataris E, Sears K, Sfetcu R, et al. Chapter 7: Systematic reviews of etiology and risk. In: Aromataris E, Munn Z, editors. JBI Manual for Evidence Synthesis. JBI (2020).

35. Adams Jr HP, Bendixen BH, Kappelle LJ, Biller J, Love BB, Gordon DL, et al. Classification of subtype of acute ischemic stroke. definitions for use in a multicenter clinical trial. TOAST. Trial of Org 10172 in Acute Stroke Treatment. Stroke. (1993) 24:35-41. doi: 10.1161/01.STR.24.1.35

36. Deeks JJ, Higgins JPT, Altman DG. Chapter 10: analysing data and undertaking meta-analyses. In: Higgins JPT, Thomas J, Chandler J, Cumpston M, Li T, Page MJ, Welch VA, editors. Cochrane Handbook for Systematic Reviews of Interventions Version 6.0. Cochrane (2019).

37. Bernasconi A, Bogousslavsky J, Bassetti C, Regli F. Multiple acute infarcts in the posterior circulation. J Neurol Neurosurg Psychiatry. (1996) 60:28996. doi: 10.1136/jnnp.60.3.289

38. Cho AH, Kim JS, Jeon SB, Kwon SU, Lee DH, Kang DW. Mechanism of multiple infarcts in multiple cerebral circulations on diffusion-weighted imaging. J Neurol. (2007) 254:924-30. doi: 10.1007/s00415-006-0397-3

39. Kang DW, Chalela JA, Ezzeddine MA, Warach S. Association of ischemic lesion patterns on early diffusion-weighted imaging with TOAST stroke subtypes. Arch Neurol. (2003) 60:1730-4. doi: 10.1001/archneur.60.12.1730

40. Sener U, Ocek L, Ilgezdi I, Sahin H, Ozcelik M, Zorlu Y. Significance of multiple acute ischemic lesions on initial diffusion-weighted imaging in stroke patients and relation of toast classification. Ann Indian Acad Neurol. (2018) 21:197-202. doi: 10.4103/aian.AIAN_487_17

41. Sorgun MH, Togay CI, Tezcan S, Yilmaz, V. Etiologic subtypes of acute multiple infarcts in more than one vascular territories. J Neurol Sci- Turk. (2016) 33:38-44.

42. Stecco A, Quagliozzi M, Soligo E, Naldi A, Cassara A, Coppo L, et al. Can neuroimaging differentiate PFO and AF-related cardioembolic stroke from the other embolic sources? Clinical-radiological correlation on a retrospective study. Radiol Med. (2017) 122:412-8. doi: 10.1007/s11547-017-0738-6

43. Sudacevschi V, Bertrand C, Chadenat ML, Tarnaud C, Pico F. Predictors of occult atrial fibrillation in one hundred seventy-one patients with cryptogenic transient ischemic attack and minor stroke. J Stroke Cerebrovasc Dis. (2016) 25:2673-7. doi: 10.1016/j.jstrokecerebrovasdis.2016.07.014

44. Vollmuth C, Stoesser S, Neugebauer H, Hansel A, Dreyhaupt J, Ludolph $\mathrm{AC}$, et al. MR-imaging pattern is not a predictor of occult atrial fibrillation in patients with cryptogenic stroke. J Neurol. (2019) 266:305864. doi: 10.1007/s00415-019-09524-5

45. Wessels T, Wessels C, Ellsiepen A, Reuter I, Trittmacher S, Stolz E, et al. Contribution of diffusion-weighted imaging in determination of stroke etiology. AJNR Am J Neuroradiol. (2006) 27:35-9.

46. Pierik R, Algra A, van Dijk E, Erasmus ME, van Gelder IC, Koudstaal PJ, et al. Distribution of cardioembolic stroke: a cohort study. Cerebrovasc Dis. (2020) 49:97-104. doi: 10.1159/000505616 
47. Maier I, Schregel K, Karch A, Weber-Krueger M, Mikolajczyk R, Stahrenberg $\mathrm{R}$, et al. Association between embolic stroke patterns, ESUS etiology, and new diagnosis of atrial fibrillation: a secondary data analysis of the find-AF trial. Stroke Res Treatment. (2017) 2017:1391843. doi: 10.1155/2017/1391843

48. Zeng Q, Tao W, Lei C, Dong W, Liu M. Etiology and risk factors of posterior circulation infarction compared with anterior circulation infarction. J Stroke Cerebrovasc Dis. (2015) 24:161420. doi: 10.1016/j.jstrokecerebrovasdis.2015.03.033

49. Tu H. Stroke and atrial fibrillation: better detection, effects on infarct evolution and outcome (Ph.D. dissertation). Melbourne, VIC, Australia (2018).

50. Diener HC, Sacco RL, Easton JD, Granger CB, Bernstein RA, Uchiyama S, et al. Dabigatran for prevention of stroke after embolic stroke of undetermined source. N Engl J Med. (2019) 380:1906-17. doi: 10.1056/NEJMoa1813959

51. Kasner SE, Swaminathan B, Lavados P, Sharma M, Muir K, Veltkamp $\mathrm{R}$, et al. Rivaroxaban or aspirin for patent foramen ovale and embolic stroke of undetermined source: a prespecified subgroup analysis from the NAVIGATE ESUS trial. Lancet Neurol. (2018) 17:1053-60. doi: 10.1016/S1474-4422(18)30319-3

52. Fuentes B, Gutirrez-Ziga R, Dez-Tejedor E. It's time to say goodbye to the ESUS construct. Front Neurol. (2020) 11:653. doi: 10.3389/fneur.2020.00653
53. Ntaios G. Embolic stroke of undetermined source: JACC review topic of the week. J Am Coll Cardiol. (2020) 75:333-40. doi: 10.1016/j.jacc.2019.11.024

54. Kamal H, Lopez V, Sheth SA. Machine learning in acute ischemic stroke neuroimaging. Front Neurol. (2018) 9:945. doi: 10.3389/fneur.2018.00945

55. Mouridsen K, Thurner P, Zaharchuk G. Artificial intelligence applications in stroke. Stroke. (2020) 51:2573-9. doi: 10.1161/STROKEAHA.119.027479

Conflict of Interest: The authors declare that the research was conducted in the absence of any commercial or financial relationships that could be construed as a potential conflict of interest.

The handling Editor declared a past co-authorship with one of the authors LC.

Copyright (c) 2020 Sharobeam, Churilov, Parsons, Donnan, Davis and Yan. This is an open-access article distributed under the terms of the Creative Commons Attribution License (CC BY). The use, distribution or reproduction in other forums is permitted, provided the original author(s) and the copyright owner(s) are credited and that the original publication in this journal is cited, in accordance with accepted academic practice. No use, distribution or reproduction is permitted which does not comply with these terms. 